Sādhanā, Vol. 19, Part 3, June 1994, pp. 487-507. (C) Printed in India.

\title{
Recent progress in dynamics and aeroelasticity
}

\author{
A R UPADHYA and KESHAB PANDA
}

Aeronautical Development Agency, P B No. 1718, Vimanapura PO, Bangalore, 560017, India

\begin{abstract}
With the emphasis on higher performance, modern aircraft designs aim at lower structural weight, aerodynamically efficient thinner configurations, and reduced or even negative stability margins augmented by automatic flight control systems. These design considerations lead to highly flexible structural designs with associated problems of dynamic and aeroelastic interactions which need to be considered at the preliminary design stage itself. Advent of advanced composites and active control techniques have given the aircraft designer the freedom to use aeroelastic interactions in an advantageous way. This paper reviews the recent research and development efforts in, the areas of aeroelastic tailoring, structural optimisation with aeroelastic constraints and aeroservoelasticity, and applications of the same in practical designs. The developments and applications in India in these areas are also highlighted.
\end{abstract}

Keywords. Aeroelasticity; aerodynamic interactions; aeroelastic tailoring; aeroservoelasticity; structural optimisation.

\section{Introduction}

The general developments in the aerospace industry during the last two decades have called for a fresh look at the role of dynamics and aeroelasticity in aerospace vehicle design. Higher performance and extended mission requirements demand designs with low structural weight, aerodynamically efficient configurations and reduced or even negative stability margins augmented by automatic flight control systems. These conflicting design requirements lead to highly flexible structural designs with associated problems of dynamic, aeroelastic and control interactions which need to be predicted and controlled at the preliminary design stage itself without undue weight penalty. Greater emphasis on the transonic flight regime presents its own special problems of flow unsteadiness and resulting structural response and flutter. Another important development that has a strong impact on design is the increasing use of advanced composite materials in primary structures such as wing and empennage. While composites offer attractive weight savings in view of their higher specific strength and stiffness properties, they also present the problem of anisotropy and resultant coupled deformation behaviours. Research studies in the past two decades have shown how the directional properties of composites could be put to advantageous use 
through aeroelastic tailoring techniques to.obtain improved aeroelastic and even aerodynamic performance without any weight penalty. On the other hand, studies on active control technology have also demonstrated possibilities of significant benefits through control and suppression of aeroelastic instabilities. However, with all the new developments, areas of overlap between structures, aerodynamics and controls in the unsteady, dynamic regime have increased leading to possibilities of aero-servoelastic (ASE) interactions. Thus an efficient aircraft design calls for an integrated design approach involving close interaction amongst aerodynamic, structural and control engineers at all stages of design. Emergence of new tools such as powerful computers and specialised application software capable of handling a multitude of design variables and parameters have made such an approach possible.

This paper briefly discusses the progress in the last decade in the following two areas: (i) aeroelastic tailoring and structural optimisation with aeroelastic constraints and (ii) aeroservoelasticity. The discussions are restricted to fixed wing aircraft. Recent work and developments in India in the above two areas are described in some detail.

\section{Aeroelastic tailoring and optimisation}

Shirk et al (1986) give a standard definition of aeroelastic tailoring as "the embodiment of directional stiffness into an aircraft structural design to control aeroelastic deformation, static or dynamic, in such a fashion as to affect the aerodynamic and structural performance of that aircraft in a beneficial way". Two technological developments in the last two decades have contributed to the evolution of aeroelastic tailoring as a design tool with great potential. These are (i) high performance fibrous composite materials and (ii) mathematical programming methods. The first has significantly increased aircraft structural design options, and the second has permitted the resulting multitude of design variables to be considered and used efficiently. Shirk et al (1986) give a comprehensive survey of the status of aeroelastic tailoring, covering the historical background, theoretical foundations and associated research studies on trends, and specific applications of the technology in design exercises. Haftka (1986) also presents developments in the United States in the application of structural optimisation under aeroelastic constraints.

Research studies reported by Shirk et al (1986) have shown aeroelastic tailoring using advanced composites to be effective in flutter control, divergence control, particularly of forward swept wings, manoeuvre load relief, improvement of control effectiveness and aerodynamic performance improvement. Figure 1 indicates the aeroelastic deformation trends to be obtained through forward or aft orientation of the primary stiffness direction in a fibre composite laminate with respect to the elastic axis and the corresponding benefits to be expected. Aft location of the primary stiffness direction results in a bend-twist coupling leading to a wash-in (increase of streamwise angle of attack along span) type of torsional deformation under an upward bending load. A wash-out type of torsion (decrease of streamwise angle of attack) is obtained under the same load with a forward location of the primary stiffness direction. It is generally seen that a design with increased aerodynamic effectiveness or improved flutter performance is poorer in terms of manoeuvre load relief and divergence characteristics. Weisshaar \& Foist (1982) developed a nondimensional stiffness cross-coupling parameter bounded between \pm 1 , in terms of the bending and torsional stiffness terms and a stiffness cross-coupling term which permitted tailoring studies to be conducted without a detailed definition of the laminate geometry. 


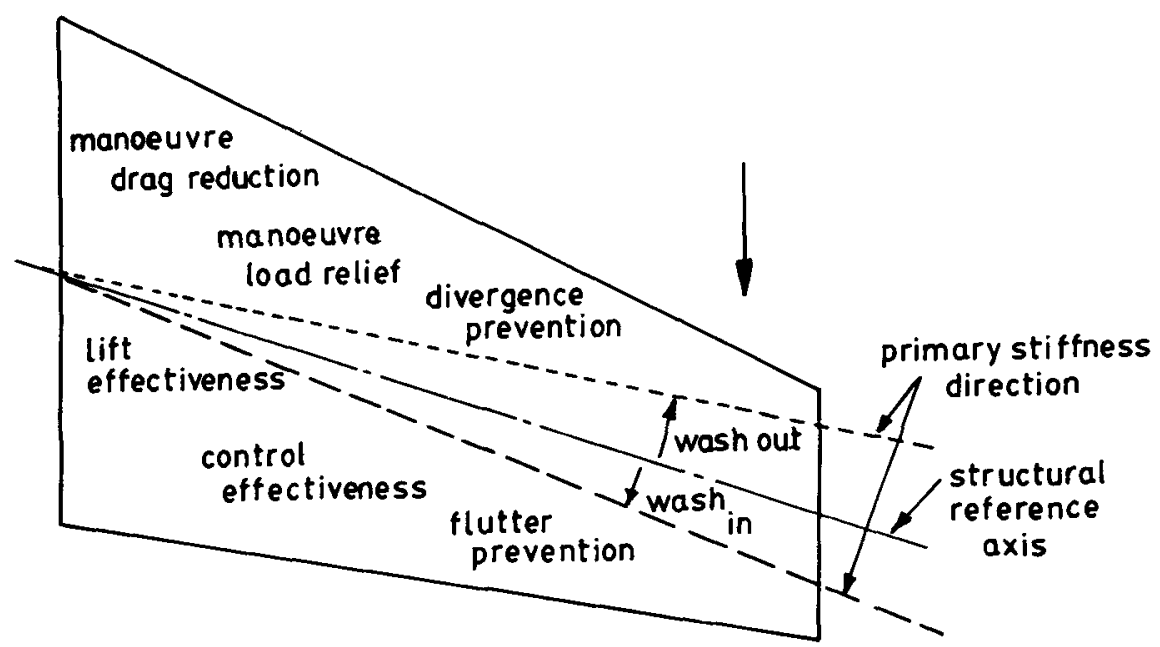

Figure 1. Benefits of aeroelastic tailoring (Shirk et al 1986).

\subsection{Software tools}

Use of composite materials, while providing the structural designer with significant design freedom, leads to a significant increase in the complexity of the design problem because of the large number of design variables involved (ply orientations, number of plies, stacking sequence etc). As a result, advanced optimisation techniques have become essential for the efficient design of composite structures. Most of the aircraft companies have developed their own in-house optimisation software to support their design activities. Of these, the following require special mention.

Wing aeroelastic synthesis procedure (TSO)-developed by General Dynamics under an US Airforce contract (McCullers \& Lynch 1974), is a preliminary design tool that employs a Ritz equivalent plate model of the wing and nonlinear programming techniques in optimisation. Minimum weight skin thickness and orientations of various plies are calculated subject to a variety of constraints including aeroelastic efficiencies, lift-curve slope and flutter and divergence speeds. The TSO program was used in several design studies for aeroelastic tailoring applications at General Dynamics (YF-16, FB-111), Boeing (KC-135, X-Wing aircraft) and MBB.

FASTOP (flutter and strength optimisation procedure) (Wilkinson et al 1977), developed by Grumman, is a finite-element-based two-step design procedure which uses the fully stressed design (FSD) criterion in the first step and a uniform flutter velocity derivative optimality criterion in the second step. The software provides a better structural idealisation as compared to TSO, but the objective function and the constraints are not as varied. FASTOP has been applied by Grumman in several design studies (FSW X-29, Israeli Lavi Fighter) and was also evaluated by Rockwell and Boeing.

ELFINI (Petiau 1990) is a finite-element-based general purpose software developed and extensively used by Dassault Aviation, France, in all its aircraft projects. It presents an integrated approach to aircraft structural analysis and design, incorporating airload computations, aeroelasticity and manoeuvre calculations, flutter analysis and structural optimisation for minimum weight based on a nonlinear projected conjugate gradient method. The design variables are the number of plies 
in each fibre direction and the constraints include aeroelastic efficiencies, natural frequencies, flutter speed and damping.

ASTROS (automated structural optimisation system) (Neill et al 1990) was developed under US Airforce contract. ASTROS is a multidisciplinary finite-element-based software system that can be used in the preliminary design of aerospace structures. It has primarily integrated existing methodologies into a unified package and, like ELFINI, provides basic static and dynamic structural analysis, steady and unsteady airloads, flutter analysis, sensitivity analysis, optimisation capabilities and aeroservoelastic response analysis. The optimisation procedure employs a method of modified feasible directions and optimality criteria methods. Optimisation constraints include flutter and divergence speeds, aeroelastic trim parameters and aeroelastic stability derivatives. Ply orientation in composite skin laminates is not a possible design variable as in TSO.

ADOP (aeroelastic design optimisation program) (Dodd et al 1990) is an interdisciplinary optimisation program for static, dynamic and aeroelastic analysis using finite element structural models. Developed by the Douglas Aircraft Company, ADOP incorporates FSD concepts, static aeroelasticity and flutter constraints in optimisation. The strategy is to perform a series of optimisation studies rather than attempt the simultaneous satisfaction of multidisciplinary constraints.

ECLIPSE (Kerr \& Thomson 1986) is a finite-element-based structural optimisation program developed by the Warton Division of British Aerospace (BAe). The program is optimality criteria based and is capable of dealing with stiffness-based criteria such as aero efficiency, roll rate, divergence speed, flutter speed, natural frequency and frequency separation. The program has been used extensively by BAe in its aircraft design projects.

A finite-element-based software called ASAT (Godel \& Schneider 1981) exists at MBB which is able to size cantilevered or free-free surface structures for flutter speed, deflection and strength constraints. CFC structures can also be treated efficiently.

\subsection{Specific applications}

Some specific applications of aeroelastic tailoring and optimisation with aeroelastic requirements in aircraft design during the past 15 to 20 years are briefly described below.

The HiMAT (highly manoeuvrable advanced technology aircraft) remotely piloted research vehicle (Lockenhauer \& Layton 1976; Shirk et al 1986), designed and built by Rockwell for NASA, was a test-bed for application of aeroelastic tailoring concepts in the lifting surfaces of a modern aircraft. The outboard wing and the canard (figure 2) of HiMAT were aeroelastically tailored to provide optimum transonic manoeuvre performance (load factor of $8 g$ at Mach 0.9 and an altitude of 20,000 ft) with minimum adverse effect on cruise performance. In the design, the aeroelastic twist distribution of the wing was maximised in order to minimise the built-in twist. The TSO program was used in the preliminary design of the vehicle. The required wing ply orientation is shown in figure 2 . The HiMAT programme demonstrated the feasibility of unconventional, unbalanced, graphite-epoxy laminates in controlling aeroelastic twist.

Feasibility of designing a forward-swept wing avoiding divergence instability through use of aeroelastic tailoring was demonstrated in the fighter-class aircraft, X-29, developed by Grumman (Hertz et al 1982). Grumman used FASTOP in the 


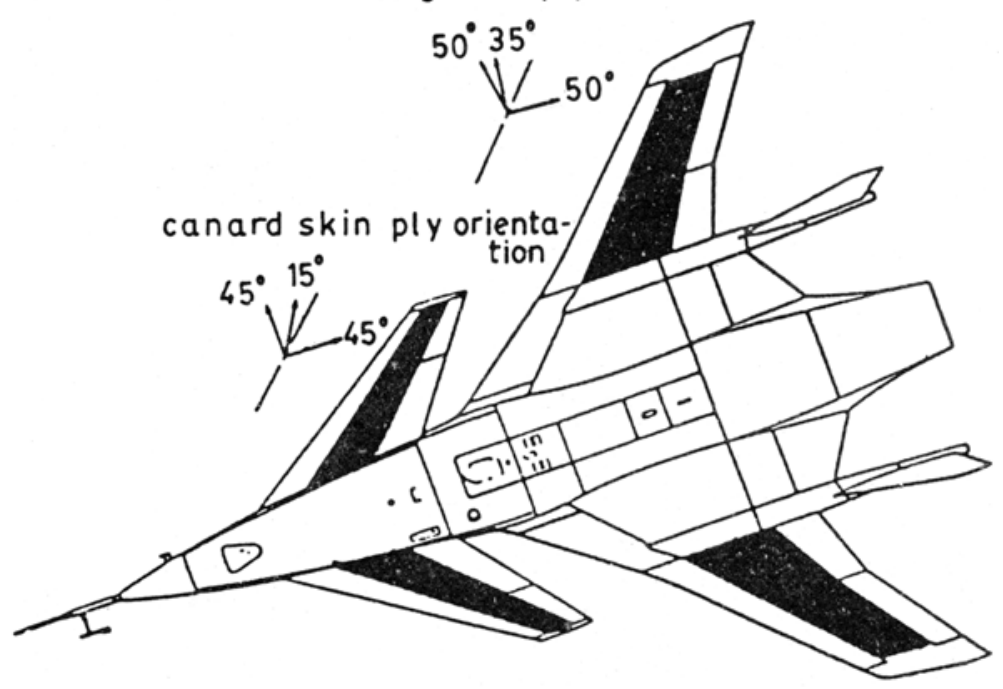

Figure 2. HiMAT remotely piloted research vehicle (Shirk et al 1986).

design and arrived at a conventional $\left(0^{\circ}, \pm 45^{\circ}, 90^{\circ}\right)$ laminate, rotated such that the primary bending plies are $9^{\circ}$ forward of the reference structural axis. This provided the necessary bend-twist coupling to minimise the inherent wash-in tendency of the forward-swept wing. A study (Ashley 1982) by Rockwell on a similar concept using TSO also led to a similar result (figure 3).

Applications of advanced optimisation techniques with composites in a combat aircraft wing \& fin using ELFINI at Dassault Aviation are presented in Petiau (1990).

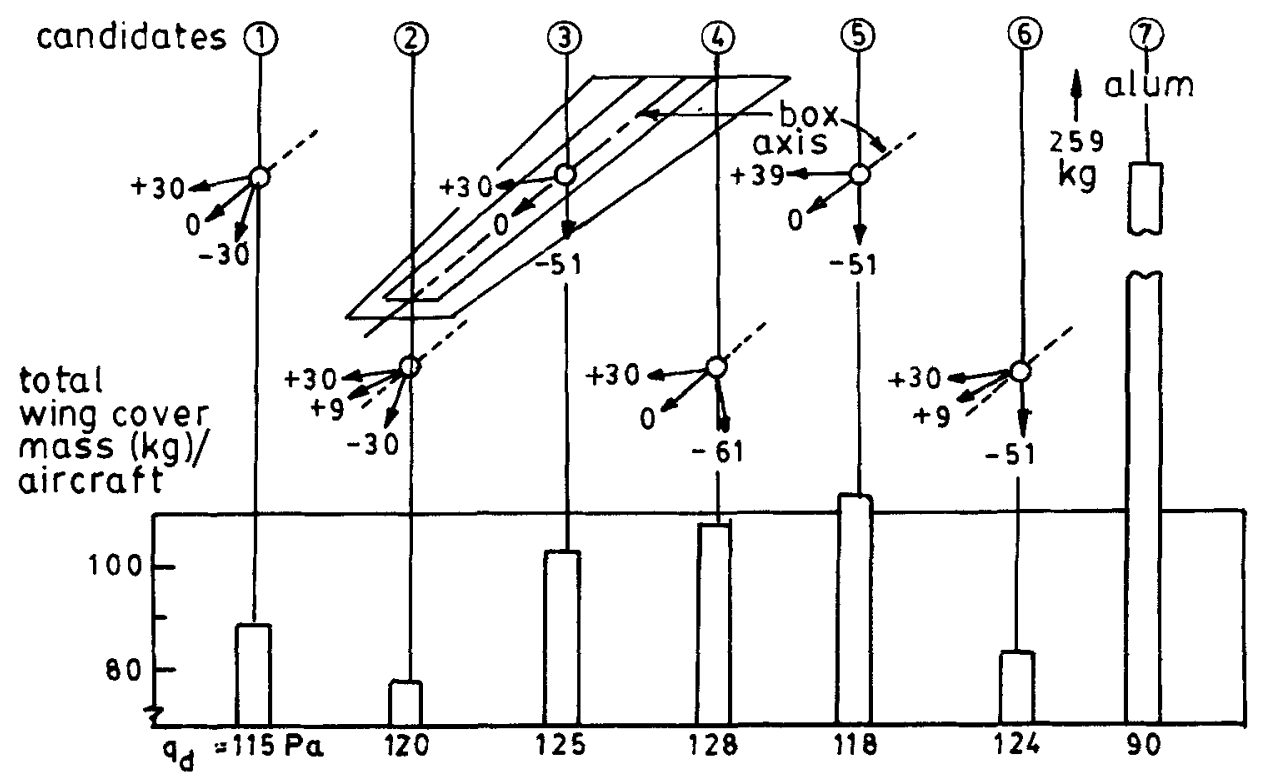

Figure 3. Rockwell FSW fighter wing skin studies (Ashley 1982) $\left(q_{d}\right.$-divergence dynamic pressure). 


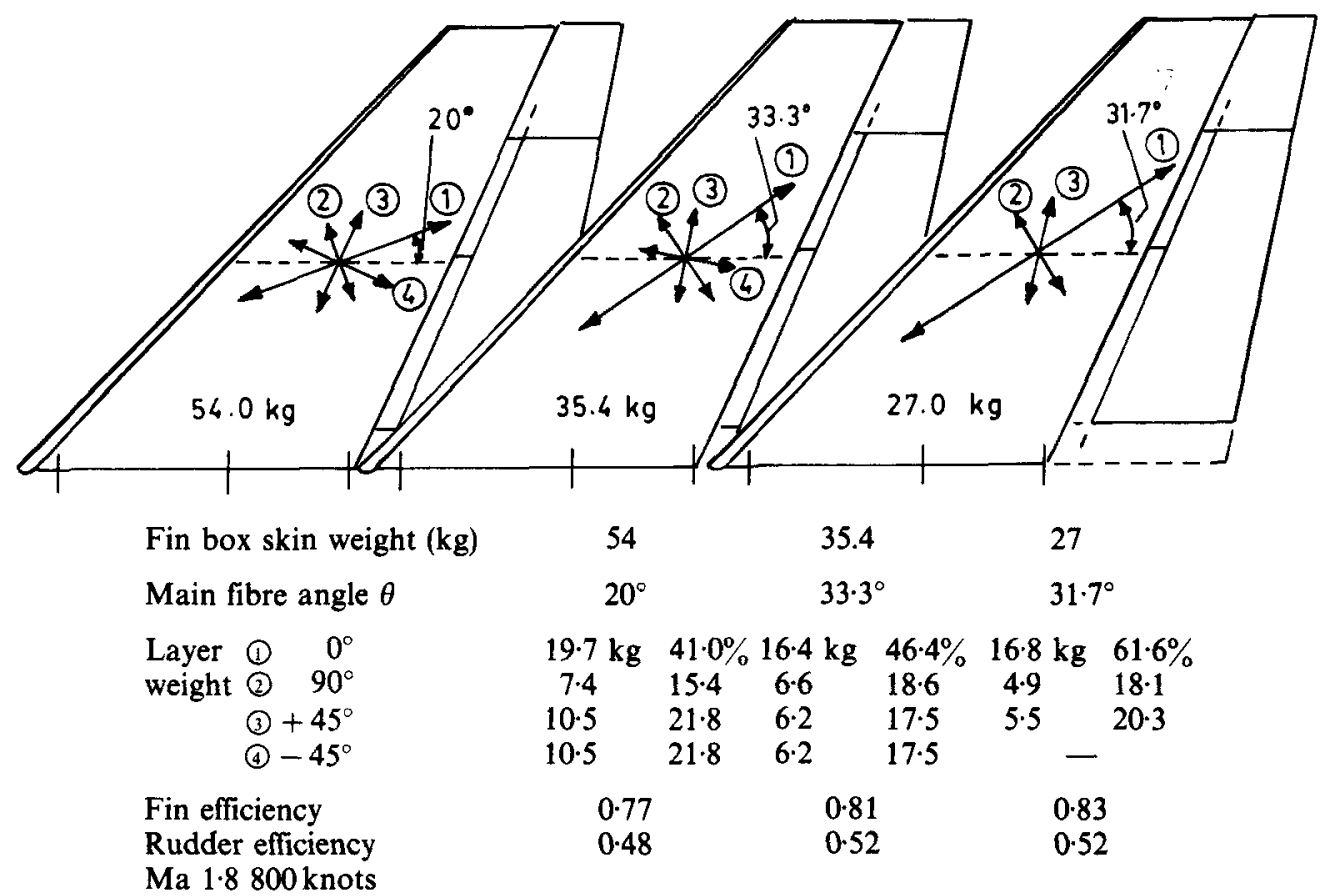

Figure 4. Fin-box skin optimisation using TSO (Schneider \& Zimmermann 1986).

The constraints considered include static aeroelasticity, flutter and dynamic frequencies.

Schneider \& Zimmerman (1986) describe the use of TSO and FASTOP at MBB in the aeroelastic design studies of the fin and wing boxes of a fighter aircraft. The fin skin optimisation results (figure 4) demonstrated significant benefits from aeroelastic tailoring. Further optimisation for flutter with FASTOP showed an $8.2 \%$ increase in flutter speed with only a $2.8 \%$ increase in the fin weight.

\subsection{Developments in India}

Efforts in recent years at the Aeronautical Development Agency towards incorporation of aeroelastic tailoring and optimisation concepts in the preliminary design phase of an advanced fighter aircraft are described in this section. The aeroelastic analysis is carried out using the general purpose computer software ELFINI.

The aircraft has a tailless delta-wing configuration with two elevons on each wing for longitudinal and roll control. The fin and rudder provide lateral stability and control. Both the wing and the fin are multi-spar configurations (figure 5) and are of predominantly CFC construction. The skins are tailored and optimised for minimum weight subject to a variety of constraints and load cases.

The aeroelastic analysis uses a finite-element structural model of the aircraft (figure 6) with about 15,000 degrees of freedom in the half symmetric model. Several basic mass distributions are defined on the model which can later be combined to create any defined design mass case.

Basic linear aerodynamic computations based on the method of singularities provide $\triangle C p$ distributions on two-dimensional aerodynamic meshes of wing and fin. 


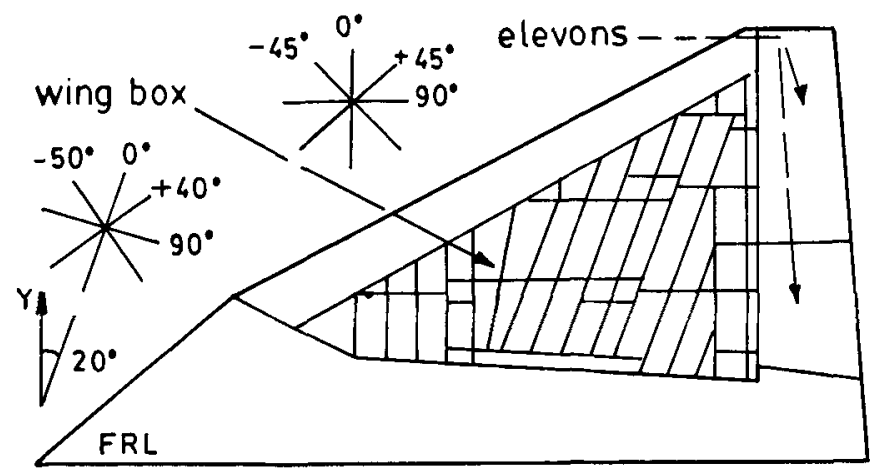

(a)

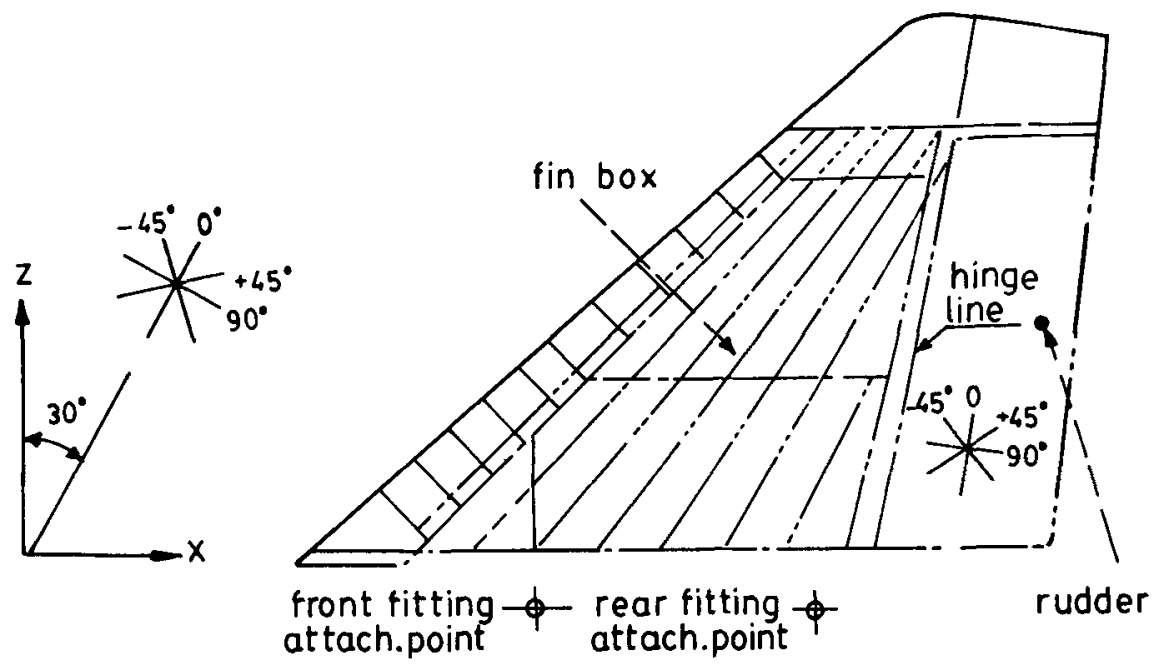

(b)

Figure 5. Fighter aircraft wing (a) and fin (b) geometry.

These distributions are corrected (or replaced) using wind-tunnel test data and results from rigorous CFD codes. Bodies such as fuselage, stores etc. are loaded by specifying force and moment resultants on aerodynamic boxes which are subsequently distributed to finite element nodes encompassed by the boxes.

The strategy for aeroelastic analysis involves a two-stage computational procedure; extensive aerodynamic and structural computations are done independently in stage 1 and stored as tables of operators which are subsequently used in stage 2 for less extensive computations of fluid-structure interactions and related aspects for specific mass cases and flight points. This is made possible with the definition of an independent computational grid on which an aerodynamic shape basis and a reduced load basis are defined and which acts as a link between finite element and aerodynamic meshes. Upadhya et al (1990a, pp. 331-7) describes in detail the aeroelastic analysis procedure adopted.

Typical results are presented in figures 7 to 9 . For an applied rigid $\Delta C p$ distribution on the fin (figure 7) for unit values of sideslip $\beta$ and rudder deflection $\delta$, at Mach 1.2 , the induced $\triangle C p$ distribution due to flexible deformation of the fin and rudder are shown in figure 8 . Aeroelastic losses occur mainly due to torsion and chordwise bending of the lifting surface and could be quite large, particularly for control aerodynamic derivatives, at supersonic Mach numbers and high dynamic pressures 


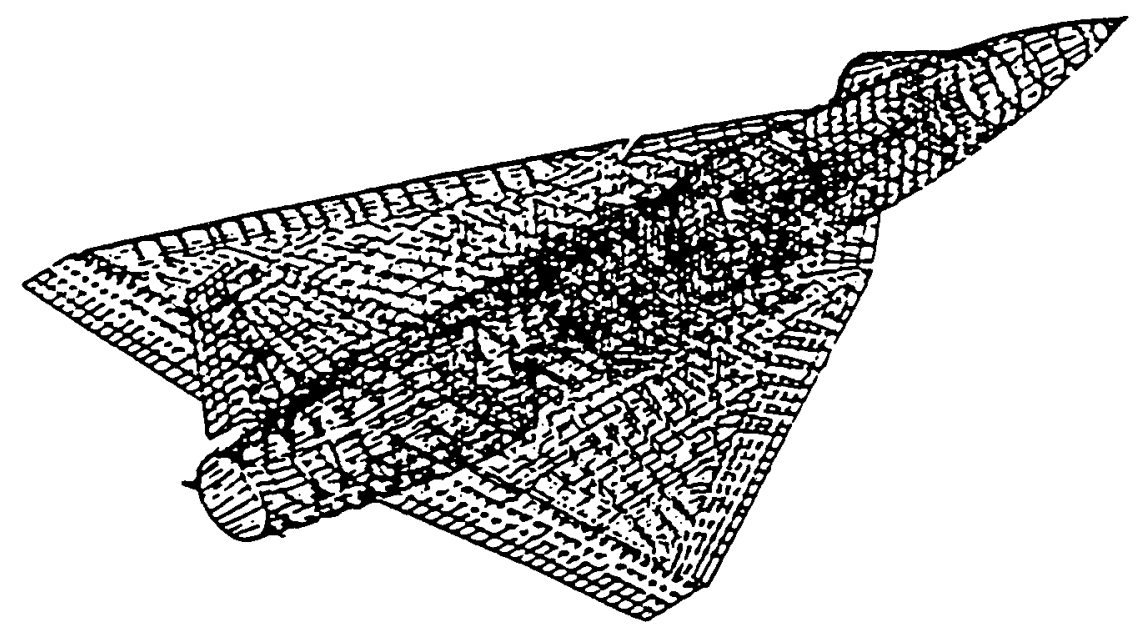

Figure 6. Finite element model.

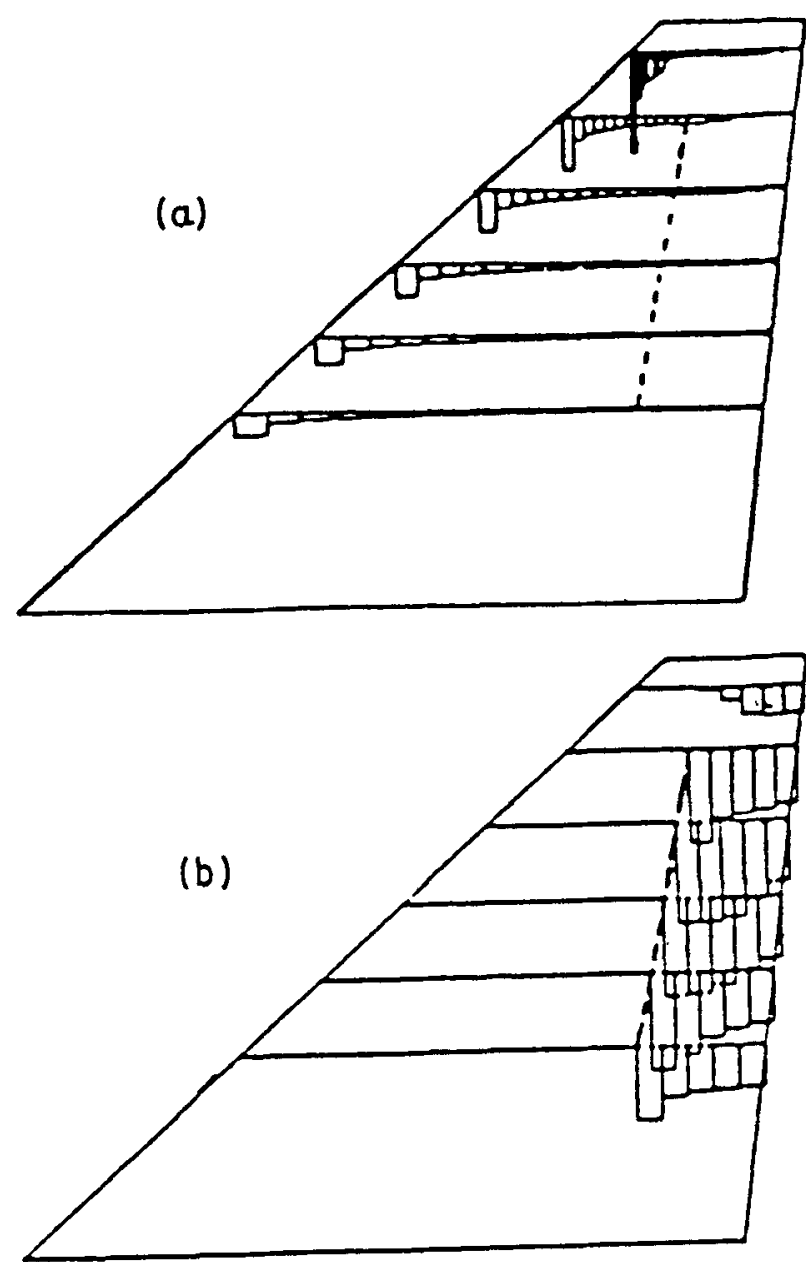

Figure 7. Applied rigid $\Delta C_{p}$ distribution on fin $(M=1 \cdot 2)$. (a) Sideslip $\beta$, (b) rudder $\delta_{r}$. 


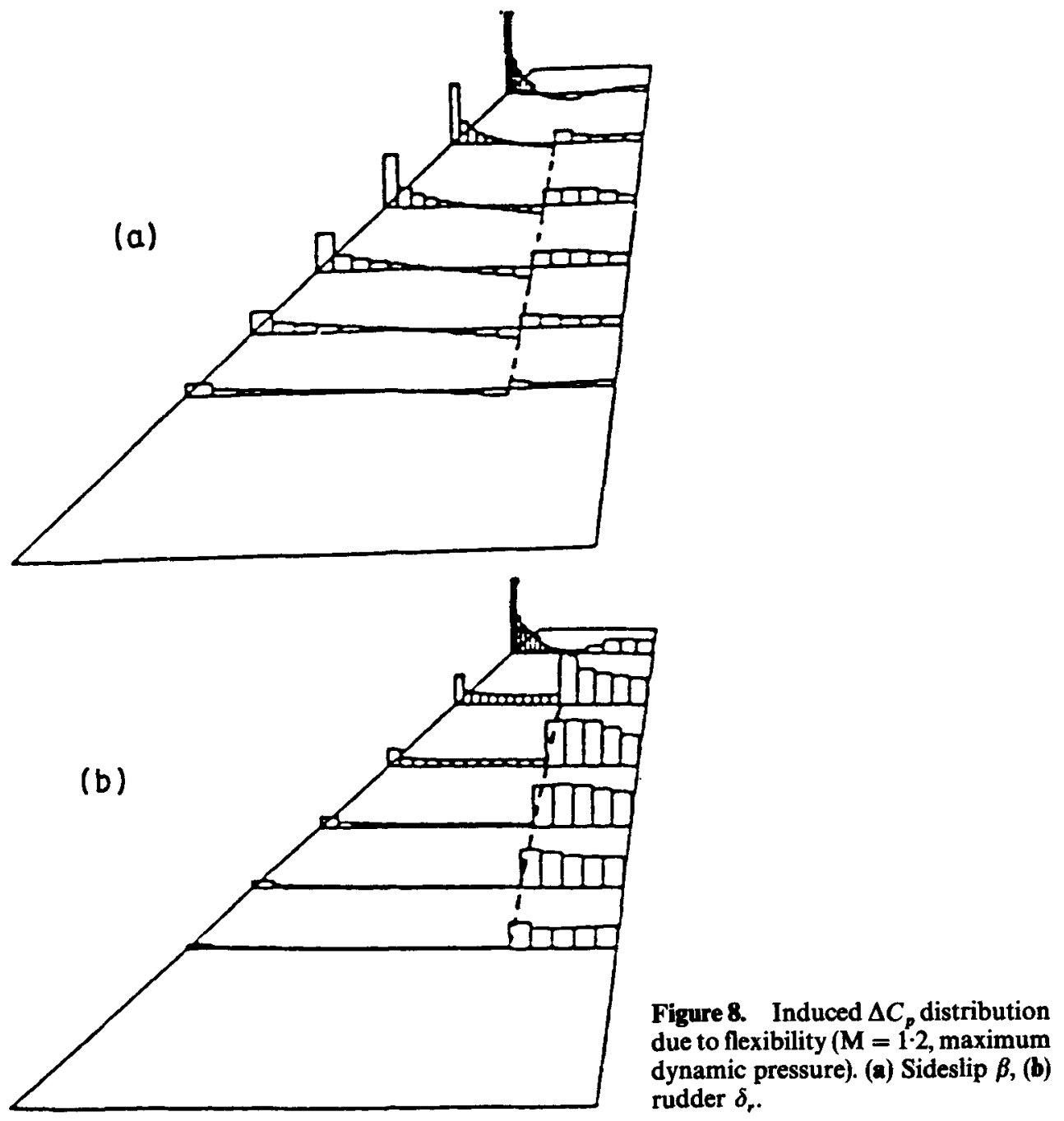

as shown in figure 9. The fly-by-wire FCS of the aircraft will define the minimum acceptable values of aeroelastic efficiency on critical control derivatives and the CFC skins are optimally designed to provide the necessary stiffness and aeroelastic.deformation coupling. Typical aeroelastic deformation shapes of the aircraft for angle of attack $(\alpha)$ and antisymmetric elevon $\left(\delta_{a}\right)$ effects are presented in figure 10.

The optimisation procedure requires definition of zones or patches on the skins (figure 11). The design variables are the thicknesses (or number of plies) in each of the four pre-defined fibre directions in the zones. The total number of design variables are 328 for the CFC wing skins and 154 for CFC fin skins.

Critical load cases involving combinations of normal load factor and roll rate/acceleration for the wing and sideslip and rudder deflection for the fin are defined for the optimisation study.

Static aeroelastic requirements in optimisation are defined as minimum acceptable values of elevon effectiveness in roll or rudder effectiveness in yaw. These parameters design the torsional stiffness of wing/fin box and also the bend-twist coupling which 


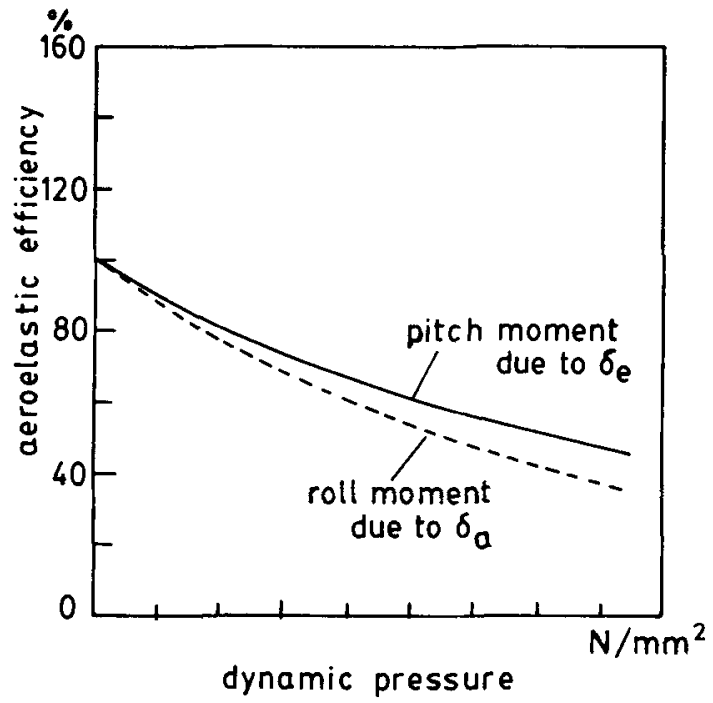

Figure 9. Aeroelastic efficiency variation.

determines the wash-in/wash-out characteristics. Other constraints considered in optimisation are failure criteria in the skins (modified Tsai-Hill criterion for composite plies), local buckling of skin panels between spars and limited technological constraints such as maximum and minimum thickness in any given direction as a ratio of total laminate thickness in a given zone. Aeroelastic constraint is an overall constraint on the structure and is independent of load cases. Details of the optimisation procedure are presented in Upadhya et al (1990b, pp. 485-90).

The results of various aeroelastic tailoring/optimisation studies on the wing are summarised below.

(1) Table 1 presents evolution of optimum weight with design constraints for a generally used value of aeroelastic efficiency parameter. These results pertain to a $0^{\circ} /+40^{\circ} /-50^{\circ} / 90^{\circ}$ lay-up.

(2) Figure 12 compares optimum weights of metallic ( $\mathrm{Al}-\mathrm{Cu}$ and $\mathrm{Al}-\mathrm{Li}$ ) and $\mathrm{CFC}$ (T300 \& T800) wing skins taking Al-Cu skin weight for roll control efficiency value $\mathrm{C}$ as reference. Value $\mathrm{B}$ corresponds to table 1 and a $5 \%$ variation on either side is considered.

It is seen that (i) for a given material, weight increase is steeper at higher values of roll efficiency as more zones come under the influence of this constraint (figure 13), (ii) the increase in weight with roll efficiency is much smaller for composites when compared to metals. This arises from the advantage of aeroelastic tailoring with composites where only the thickness in a required direction is increased to meet aeroelastic requirements.

(3) Table 2 compares relative weights of wing skins for various types of lay-ups. It is seen that unbalanced $0^{\circ} \%+45^{\circ} /-45^{\circ} / 90^{\circ}$ lay-up gave the minimum weight. Balanced lay-up resulted in a $31 \%$ weight penalty. Comparison of weights of lay-ups 1, la, 2 and 3 showed that orienting the $0^{\circ}$ fibres along the inclined spars is the optimum arrangement. The results also showed that in lay-up 1 , the $+45^{\circ}$ direction was the most predominant one, accounting for $43.6 \%$ of the weight, followed by $-45^{\circ}(28.2 \%)$, $90^{\circ}(15.6 \%)$ and $0^{\circ}(12.6 \%)$ layers. In the case of balanced lay-up, both $\pm 45^{\circ}$ layers shared $37.2 \%$ of the weight. It is thus clear that while both $\pm 45^{\circ}$ layers contribute towards torsional stiffness, the predominant $+45^{\circ}$ layers in lay-up 1 also provide 

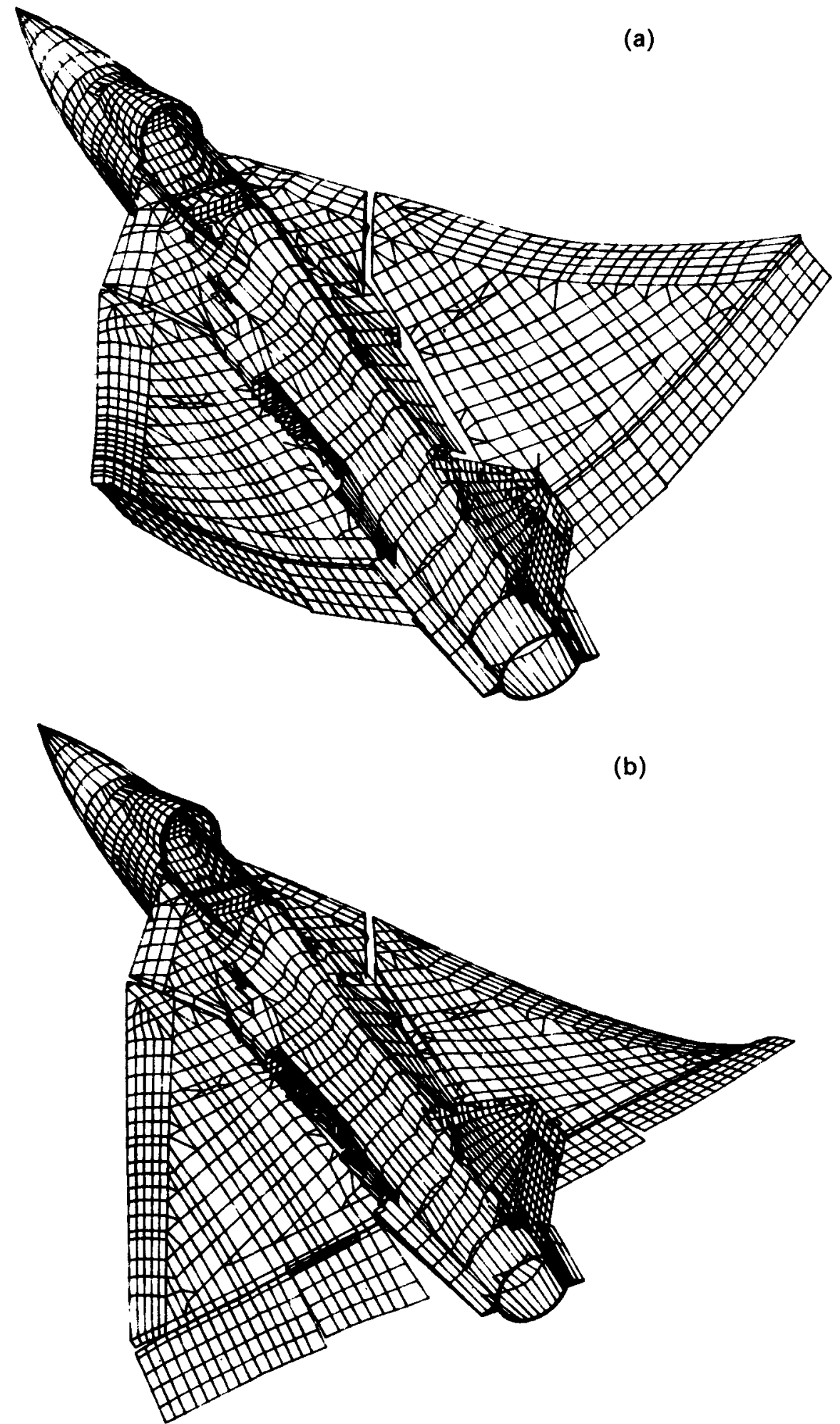

Figure 10. Aeroelastic deformation shapes, $\alpha(a)$ and $\delta_{a}(b)$ effects. 


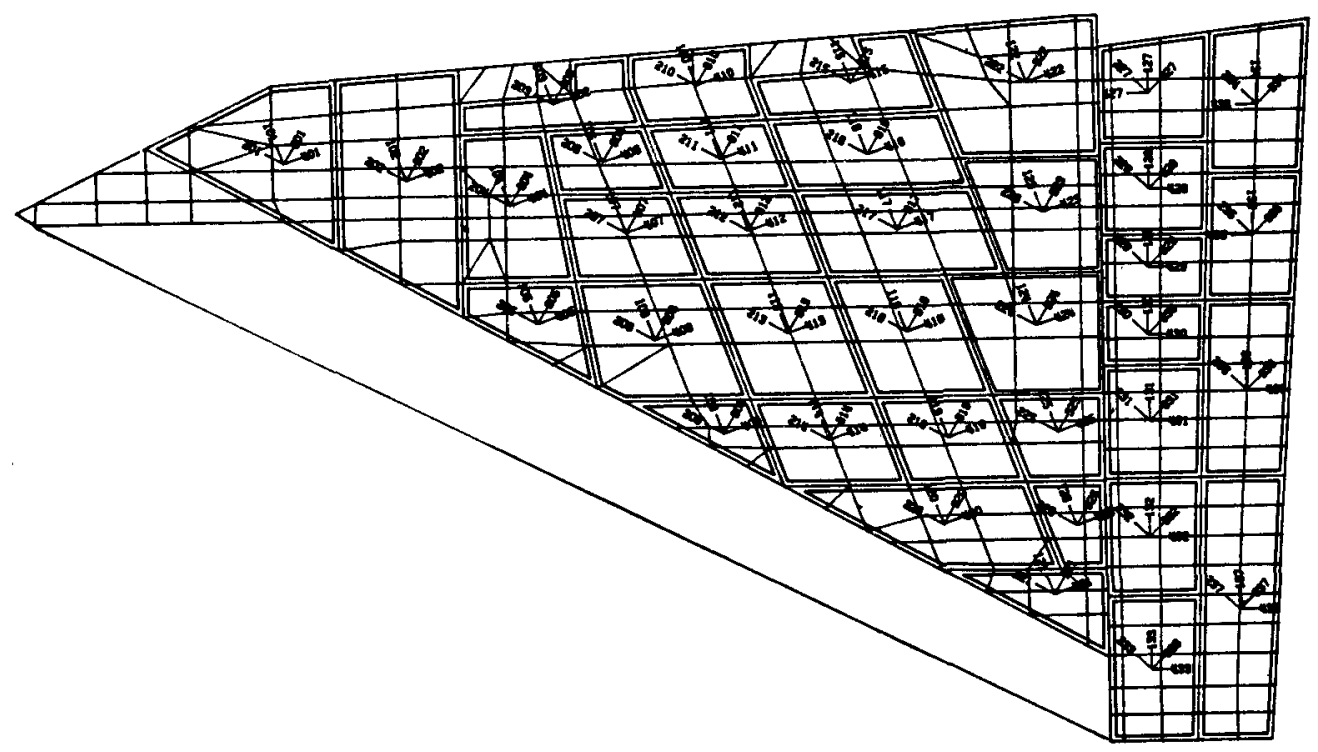

Figure 11. Optimization zones on wing skins.

the required twist-bend coupling, causing a reduced nose-down twist (i.e., effective reduction in angle of attack) under an up-load on the elevon, leading to a lower weight structure for a given elevon effectiveness. In other lay-ups also, it was seen that the $+\theta$ layers were the most predominant.

(4) The wing tip displacements in a critical load case for design case $\mathrm{B}$ for the $\mathrm{Al}-\mathrm{Cu}$, $\mathrm{Al}-\mathrm{Li}, \mathrm{T} 300$ and T800 material designs were 179, 176, 210 and $201 \mathrm{~mm}$, respectively. The higher wing tip deflections in the composite wing are again due to the tailoring effect where the bending stiffness is not increased in the same proportion as the torsional stiffness like in metal wings.

Aeroelastic analysis and optimisation studies on the fin (Kamal et al 1989) showed trends similar to that seen on the wing with the $+45^{\circ}$ layers being predominant. This design results in a coupled bending of the fin due to torsional load from the rudder, which induces a streamwise incidence opposed to the twist due to torsion, thus reducing the losses in the yaw moment effectiveness of the rudder due to flexibility. Another interesting observation was that rotating the entire lay-up, such that the

Table 1. Evolution of optimum weight. Lay-up: $0^{\circ} /+40^{\circ} /-50^{\circ} / 90^{\circ}$ on wing-box skin.

\begin{tabular}{llc}
\hline No. & \multicolumn{1}{c}{ Design constraints } & $\begin{array}{c}\text { Weight } \\
\text { (ratio) }\end{array}$ \\
\hline 1. & $\begin{array}{l}\text { Composite material - stress (failure criteria } \\
\text { constraints only) }\end{array}$ & 0.46 \\
2. & Stress + buckling constraints & 1.0 \\
3. & Stress + buckling + aeroelasticity & 1.25 \\
4. & 3 + Technological constraints & 1.31 \\
5. & Aeroelastic constraint only & 0.90 \\
6. & Aluminium alloy skin (comparable with 4) & $2 \cdot 25$ \\
\hline
\end{tabular}




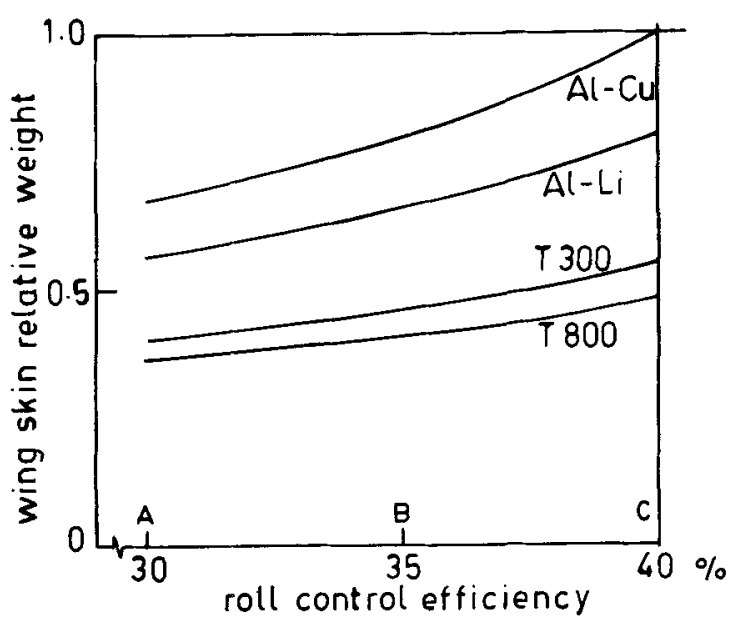

Figure 12. Comparison of optimum design wing skin weights.

angle between $0^{\circ}$ fibres and the $Z$-axis varied in the range $15^{\circ}$ to $35^{\circ}$, showed minimum skin weight $\left(9 \%\right.$ weight saving as compared to the $30^{\circ}$ case) for the $15^{\circ}$ orientation. This is most likely due to the fact that the predominant $+45^{\circ}$ layers provide relatively more direct bending stiffness for this orientation, resulting in less induced spanwise incidence due to direct bending of the elastic axis due to sideload on the rudder. The most optimum fibre orientation is one which is a compromise between the coupled bending and direct bending effects which induce spanwise incidence in opposite directions.

The study also revealed that the fin skin weight is very sensitive to the aeroelastic
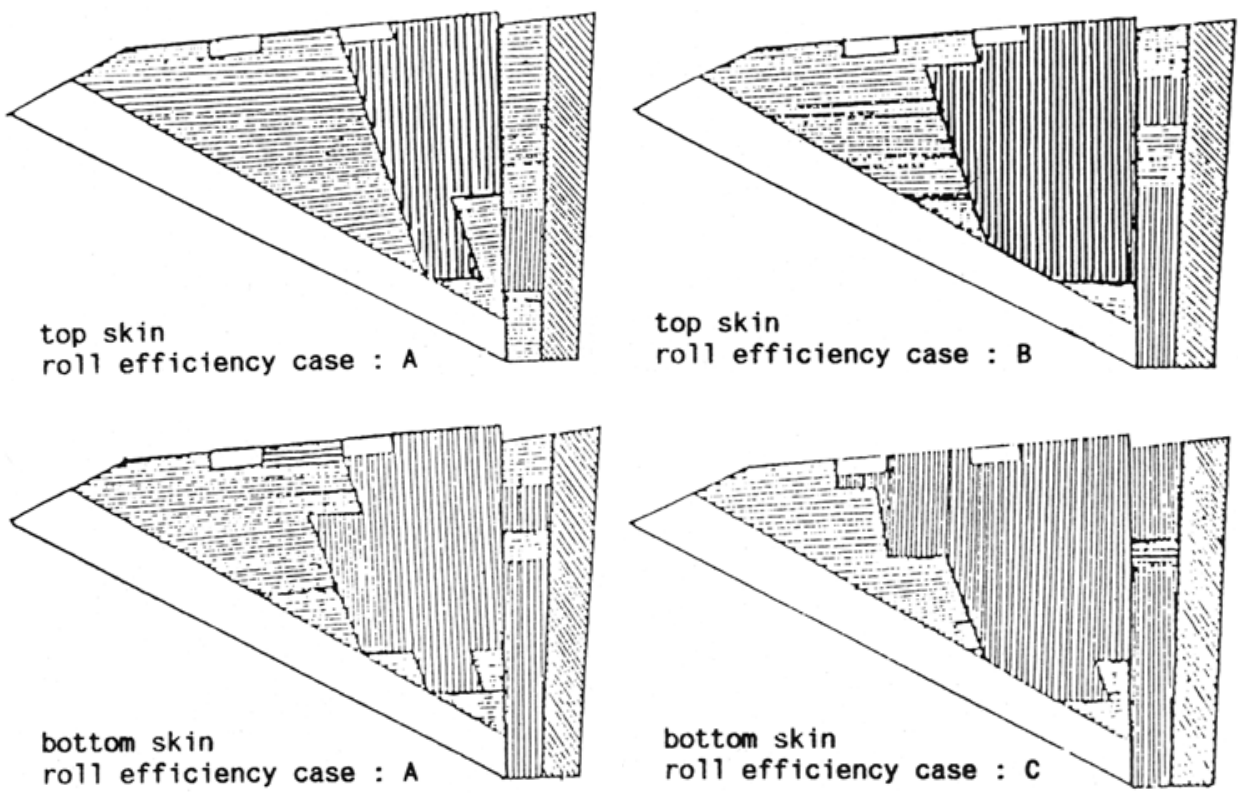

buckling

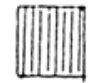

aeroelasticity

technology

Figure 13. Optimisation constraint influence zones. 
Table 2. Relative weight comparison.

\begin{tabular}{lc}
\hline Wing-box skin lay-up & Relative weight \\
\hline $0^{\circ} /+45^{\circ} /-45^{\circ} / 90^{\circ}$ & $1 \cdot 00$ \\
$0 /+45 /-45 / 90$ (balanced) & $1 \cdot 31$ \\
$10 /+45 /-45 / 90$ & $1 \cdot 20$ \\
$-10 /+45 /-45 / 90$ & 1.25 \\
$0 /+40 /-40 / 90$ & 1.23 \\
$0 /+40 /-50 / 90$ & $1 \cdot 18$ \\
$0 /+50 /-40 / 90$ & 1.29 \\
$0 /+50 /-50 / 90$ & 1.25 \\
$0 /+22.5 /-67.5 / 90$ & 1.65
\end{tabular}

Note: Reference axis for lay-up is inclined $20^{\circ}$ aft of $Y$-axis (figure 5 ).

efficiency parameter. A requirement of 5\% increase in supersonic yaw moment effectiveness of rudder resulted in a fin skin weight increase of nearly $36 \%$.

\section{Aeroservoelasticity}

For the modern aircraft, use of an efficient flexible structure is possible with the availability of composite materials and modern design and analysis techniques, aided by large capacity, high speed computers and sophisticated structural testing methods. As the structure became more flexible, the structural frequencies have come down. At the same time advances in aircraft design have necessitated expansion of the role played by flight control systems (FCS). The aircraft is designed to be highly manoeuvrable, light, and more agile. For such an aircraft the flight control system is not just used for the control of the flight path, but also to meet the above design requirement. Thus the role of FCS has been expanded. High authority control systems, utilizing multiple blended feedbacks, are used to provide tailored aircraft response to meet mission requirements. In addition, the flight control system is used to provide artificial stability for statically unstable aircraft, reduce fatigue damage to the structure from gusts and manoeuvres, suppress flutter modes and improve ride quality. This active control technology helps to reduce structural weight of the aircraft, and provides a better aerodynamic design. With the introduction of high gain, faster response flight control systems to meet the above active control requirements, the control frequency bandwidth has increased. Further, as the frequency of operation of the control system increases, it excites the aircraft structure at higher frequencies, as a result of which the reduced frequency characterising the flow unsteadiness becomes significantly large indicating that unsteady aerodynamic effects need to be accounted for. The reduction in structural frequencies and coupled increase in the control system frequency bandwidth with the presence of unsteady aerodynamics leads to a new dynamic interaction problem which is defined as aeroservoelasticity (ASE). Thus, ASE is a multidisciplinary technology dealing with the interaction of the aircraft's flexible structure and the unsteady aerodynamic forces resulting from the motion of the aircraft with its flight control system. Detailed and complex mathematical models incorporating the effects of these technical disciplines are required to accurately predict ASE interactions and to design active control systems for flexible vehicle application. 


\subsection{ASE encounters}

Several instances of ASE encounters for a variety of research, development, prototype and production aircraft are documented in literature. Felt et al (1979) have discussed in depth ASE encounters for the following aircraft:

B-36: An ASE instability was induced by the autopilot of B-36. For this aircraft the sensor package had been located in the tail gunner's compartment and significant body bending (flexible) motion had been picked up by the sensor. The solution was to move the sensor package to a position of relatively small body bending motion.

YF-16: This aircraft experienced two separate ASE instabilities during early flight tests. Initially these instabilities were not identified by analysis, because the analysis was carried out for high Mach number, low altitude flight conditions which were the most critical for flutter. However, a critical interaction occurred at a high subsonic Mach number, which was not considered in analysis. Later ASE analysis at the proper flight condition predicted the ASE instabilities which matched with the flight test results.

YF-17: YF-17 experienced two ASE instabilities, which were predicted during the analysis stage and verified with flight test results. Parametric ASE analysis of ground test and flight test interactions were carried out to select final notch filters for the flight control system.

B-52: The CCV ride quality system tested on a B-52 encountered servoelastic oscillations on ground due to local structural vibration of bulkheads or support beams. As-the local structural details were not included in the dynamic mathematical model, this interaction was not identified by the analysis. By changing accelerometer mounting, the oscillations were eliminated.

F-4: ASE instability was encountered in modified F-4 aircraft during a sideslip manoeuvre in a gear down, flap down configuration. A resonance in the pitch axis was encountered. The instability occurred at $23 \mathrm{~Hz}$, which was close to both the flap rotation mode and the stabilizer rotation mode frequencies. The instability mechanism was initiated by a flap buffet which fed into a pitch rate gyro located in the left wing root just forward of the flap. The problem was solved by additional filtering in the pitch axis.

$F-16$ and $F-18$ : These aircraft also exhibited adverse dynamic interactions between the airframe aeroelastic characteristics and their flight control systems. Flight control modifications were carried out to solve this interaction problem.

\subsection{General formulation of ASE problem}

Very few references are available in literature on ASE formulation. Noll et al (1989) present activities in the area of ASE at the NASA Langley Research Centre. They have discussed different modelling and analysis techniques for the prediction of ASE interactions. Suryanarayan et al (1992) have discussed in detail various analysis steps required to be carried out for ASE analysis. The steps are given starting from structural, aerodynamic and control system modelling up to identification of ASE interations in 
the flight envelope. The steps are as below.

(i) Normal mode analysis of the aircraft structure for a given mass case (mode shapes, frequencies, modal mass and modal stiffness computation).

(ii) Calculation of modal unsteady airloads as a function of reduced frequency and Mach number for harmonic motion.

(iii) Representation of modal unsteady airloads in the time domain for arbitrary motion.

(iv) Dynamic modelling of the actuator assemblies.

(v) Development of a flexible aircraft dynamic model taking into account the effects of elastic modes and unsteady airloads along with the actuator model (state-space model of flexible aircraft).

(vi) Open loop poles and zeros computation, formation of open loop transfer function for the flexible aircraft.

(vii) Calculation of open loop frequency response at the sensor location for a sinusoidal input to the actuator from the state-space model as well as from the open loop transfer functions.

(viii) Representation of control system dynamics in terms of transfer functions.

(ix) Development of closed loop aircraft description taking all the feedback element dynamics into account.

(x) ASE stability analysis for various flight points in the flight envelope.

The flexible aircraft state-space model as given in step ( $v$ ) is used for active control design applications.

\subsection{ASE analysis tools}

The following general purpose analysis programs are documented in the literature for carrying out ASE analysis.

STARS (Gupta et al 1989): Structures, aerodynamics and related aeroservoelastic system analysis is a general-purpose structural analysis program that has a complete aeroservoelastic analysis capability. In the package, Pade and least squares approximation methods are available for time domain representation of unsteady aerodynamics and it is used for open and closed loop aeroservoelastic controls analysis.

ADAM and ADAM-2.0 (Noll et al 1986) (Analog and digital aeroservoelasticity method): This program combines the technologies of unsteady aerodynamics, multi-input/multi output (MIMO) controls and structural dynamics into an interactive analysis package.

ISAC (Interaction of structures, aerodynamics and control) (Sallee 1990): All the active control problems at NASA Langley are analysed using ISAC. The package has 3 methods for performing aerodynamic approximation and uses optimal method for control law synthesis.

ASEPACK, Aeroservoelasticity package (Suryanarayan et al 1992): This program has been developed at the Aeronautical Development Agency. The program takes structural dynamics and frequency domain unsteady aerodynamics input from the general purpose finite element package ELFINI. The package has been developed on similar lines to STARS. Various in-house developed new analysis techniques, namely, modified rational function approximation (RFA) for time-domain representation of unsteady aerodynamics and low-order aircraft plant model design for the use of flight 


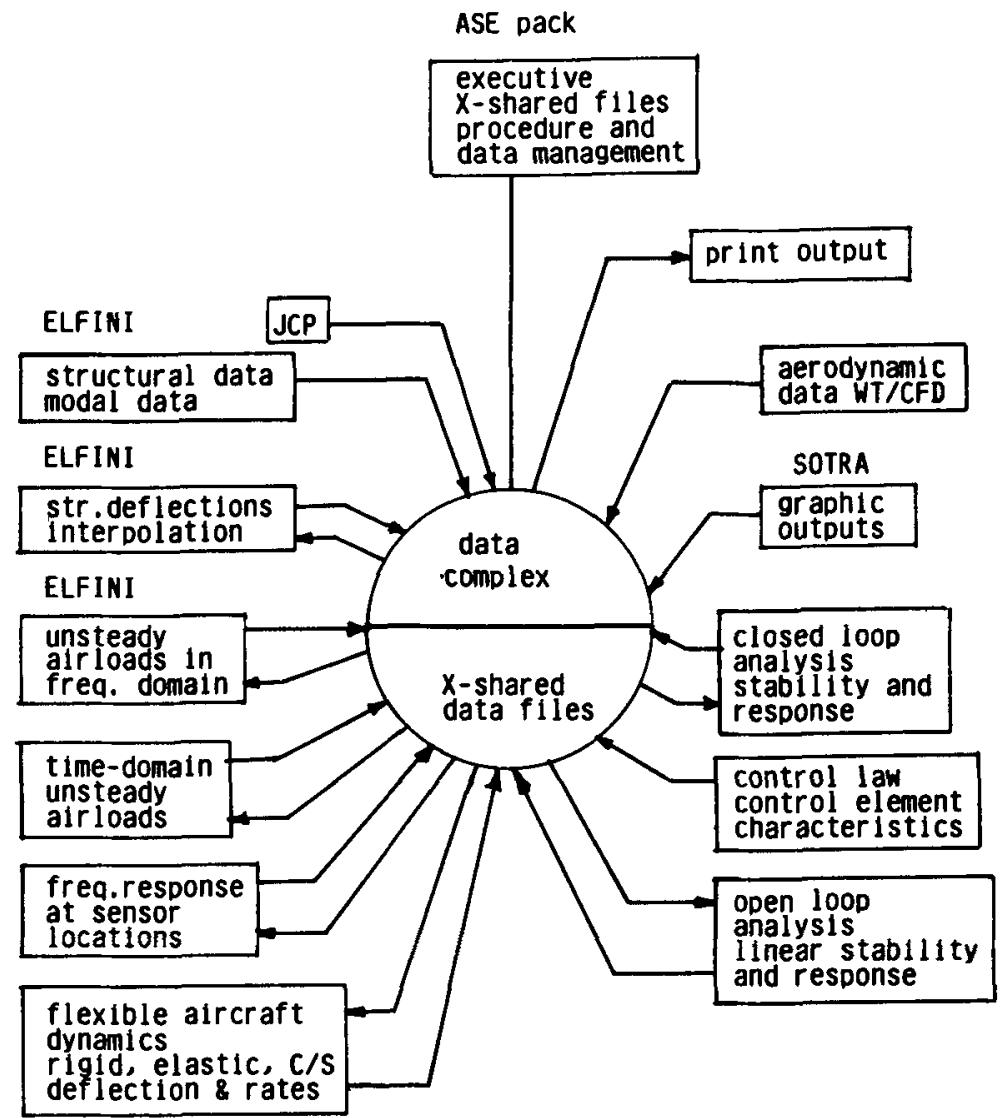

Figure 14. ASEPACK block diagram (Suryanarayan et al 1992).

Table 3. Comparison of ASEPACK and STARS programs.

\begin{tabular}{lll}
\hline Capability & ASEPACK & \multicolumn{1}{c}{ STARS } \\
\hline O/L and C/L S-plane stability analysis & Yes & Yes \\
Loop closure with feedback elements & Yes & Yes \\
Bode plots of O/L and C/L frequency response & Yes & Yes \\
Maximum state variables & No limit & 100 \\
Aerodynamic lag terms & No limit & 4 \\
Time history analysis & Yes & Yes \\
Actuator representation & Fourth order & Fourth order \\
Sensor dynamics & Yes & Yes \\
Anti-aliasing filter & Second order & First order \\
Notch/shaping filter & Yes & Yes \\
Subsonic/supersonic & Yes & Yes \\
O/L and C/L Z-plane stability analysis & Yes & Yes \\
Time domain representation & Pure lag & Conventional \\
Reduced order plant representation & Yes & No \\
\hline
\end{tabular}


control-law design and simulation studies are implemented in this package. Figure 14 shows the ASEPACK block diagram. Table 3 shows a comparison of the capabilities of the ASEPACK and STARS programs.

\subsection{Application of ASEPACK to a typical fighter aircraft}

The ASE analysis package is used for analysis of a typical fly-by-wire fighter aircraft configuration. Figure 15 shows the first wing bending and torsion modes, first fuselage bending mode, and also a line representation of fuselage deformation in the first five symmetric modes. Figure 16 shows the accuracy of the time-domain representation of a typical aerodynamic coefficient and figure 17 shows the open-loop normal acceleration (both amplitude and phase) response of the flexible aircraft plant at the sensor location with the rigid and flexible contributions and the total response shown separately. For the present analysis two rigid body modes (heave and pitch) and the first four symmetric elastic modes covering wing bending and twisting, and fuselage bending are considered. Both open-loop and closed-loop stability and frequency response results are generated for a typical flight condition.

(a)

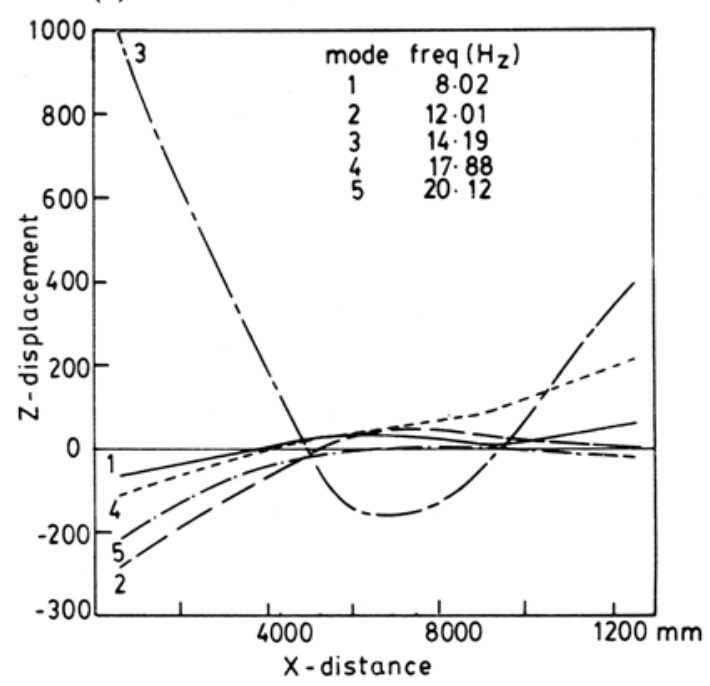

\section{(c)}

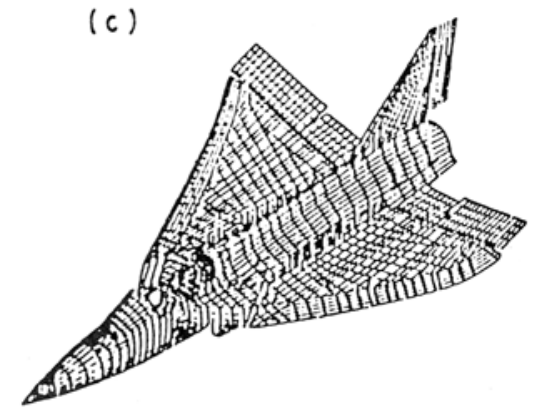

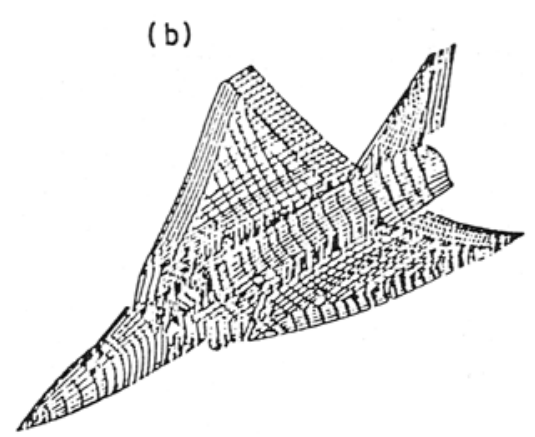

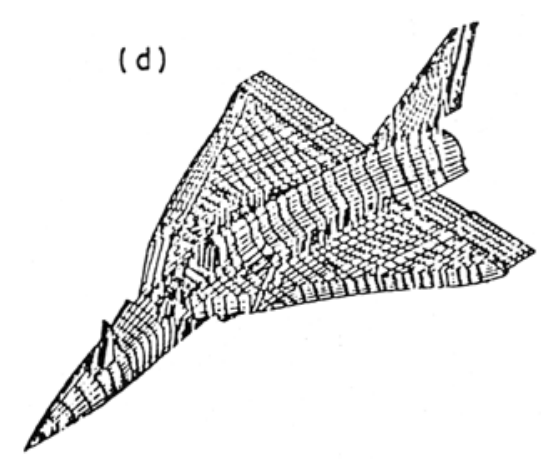

Figure 15. Free vibration mode shapes (Suryanarayan et al 1992). (a) Fuselage symmetric mode shapes; (b) wing bending; (c) wing torsion; (d) fuselage bending. 


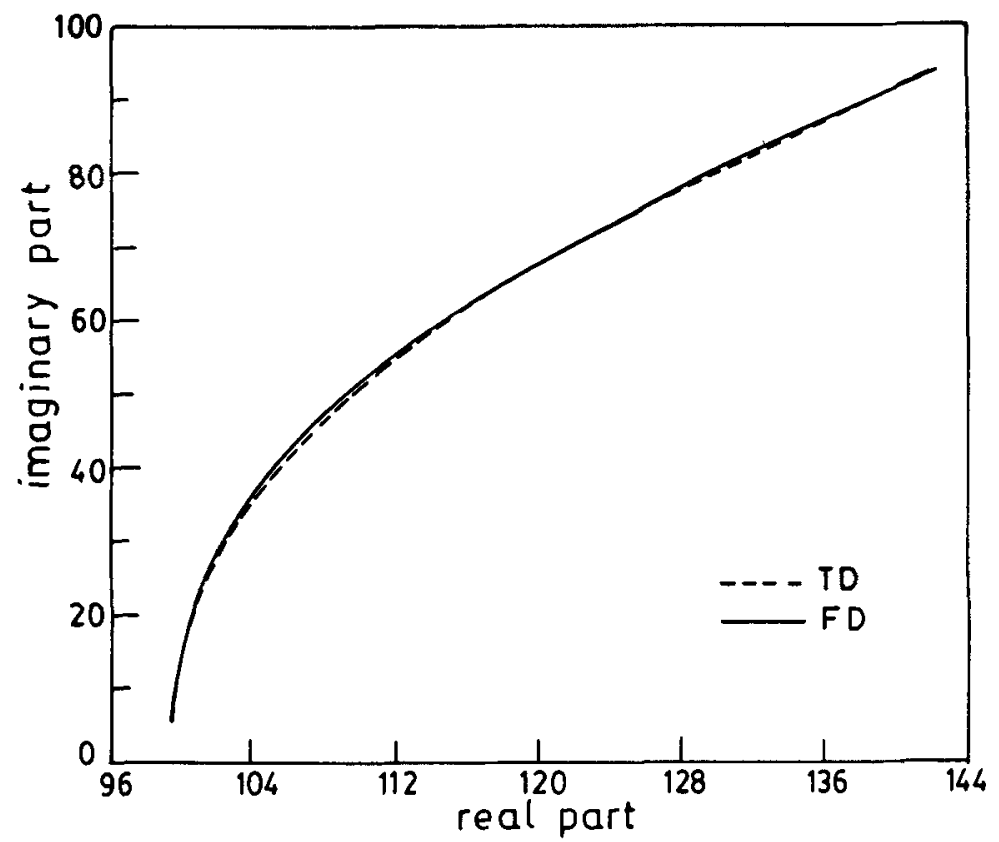

Figure 16. Frequency domain (FD) and time domain (TD) representation of $C_{L \alpha}$ (Suryanarayan et al 1992).

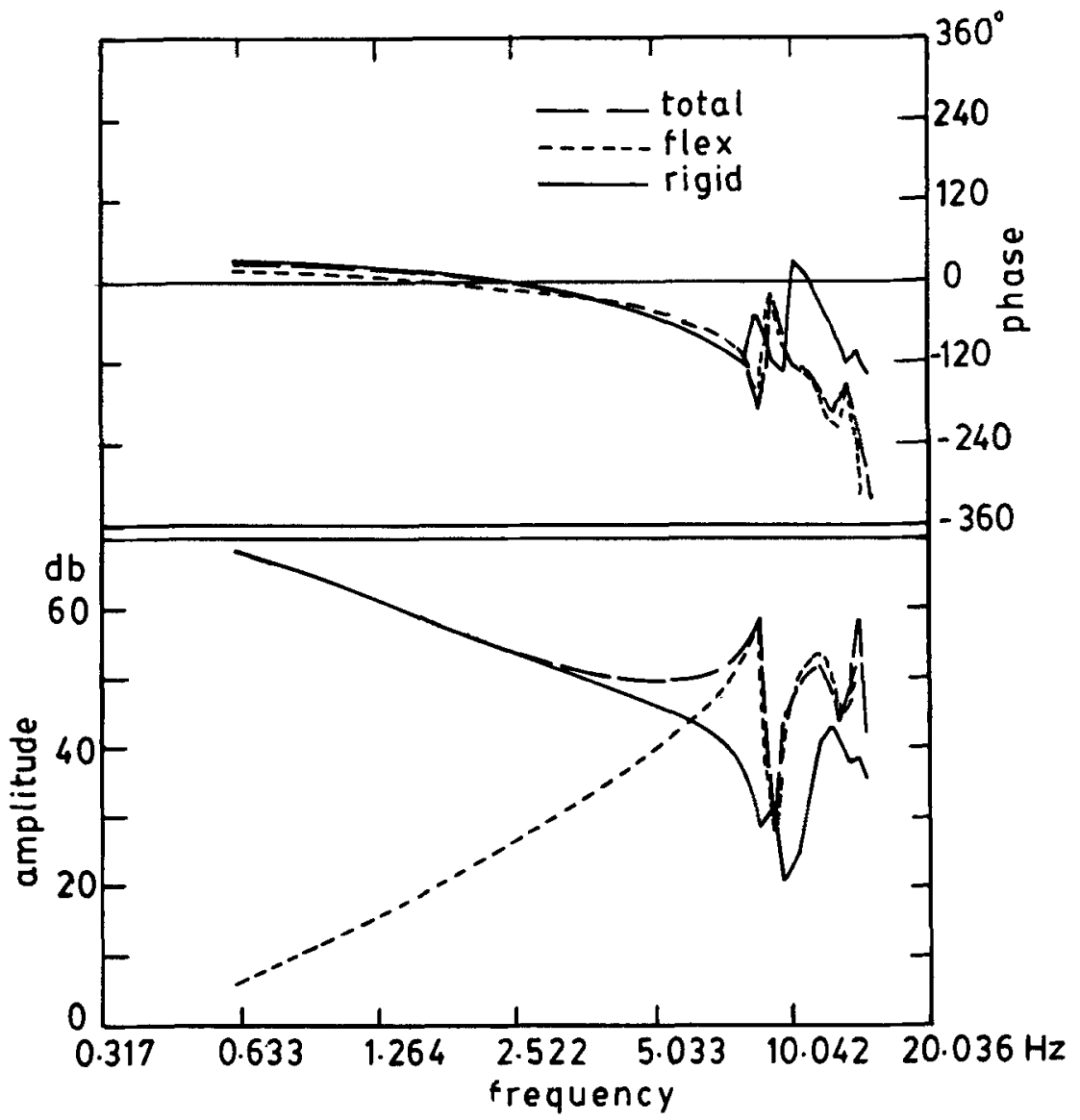

Figure 17. Open loop frequency response results (Suryanarayan et al 1992). 


\section{Conclusions}

In the preceding sections, recent progress in the areas of aeroelastic tailoring, structural optimisation with aeroelastic requirements and aeroservoelasticity are discussed. Developments and applications of the above technologies in India are highlighted.

So far aeroelastic tailoring and active control technology have progressed independently. However, the aeroelastic benefits to be derived from the two techniques have their own limits. Effective integration of structural stiffness tailoring and active controls through simultaneous design of a structure and control law holds great promise (Livne et al 1990; Dracopaulos \& Oz 1992). It can be expected that they will merge into a single design tool within the next decade.

The authors are grateful to Dr Kota Harinarayana for encouragement.

\section{References}

Ashley H 1982 On making things the best - Aeronautical uses of optimisation. J. Aircr. 19: $5-28$

Dodd A J, Kadinka K E, Loikkanen M J, Rommel B A, Sikes G D, Strong R C, Tzong T J 1990 Aeroelastic design optimisation program. J. Aircr. 27: 1028-1036

Dracopoulos T N, Oz H 1992 Integrated aeroelastic control optimisation of laminated composite lifting surfaces. J. Aircr. 29: 280-288

Felt L R, Huttsell L J, Noll T E, Cooley D E 1979 Aeroservoelastic encounters. J. Aircr. 16: $477-483$

Godel H, Schneider G 1981 Application of a structural optimisation procedure for advanced wings. AIAA-R-691

Gupta K K, Brenner M J, Voelker L S 1989 Integrated aeroservoelastic analysis capability with X-29A comparisons. J. Aircr 26: 84-90

Haftka R T 1986 Structural optimisation with aeroelastic constraints: A survey of Us applications. Int. J. Vehicle Design 7: 381-392

Hertz T J, Shirk M H, Ricketts R H, Weisshaar T A 1982 On the track of practical forward swept wings. Astronaut. Aeronaut. 20: 40-52

Kamal K, Srinivasan K, Panda K 1989 Preliminary optimisation study of a fighter aircraft fin. ADA/060200/040/89

Kerr R I, Thompson D 1986 Automated structural optimisation at Warton. Proc. 15th Congress of ICAS (eds) P Santini, R Staufenbiel Paper ICAS-86-3.1.3 (New York: AIAA) 1: 130-134

Livne E, Schmit L A, Friedmann P P 1990 Towards integrated multidisciplinary synthesis of actively controlled fibre composite wings. J. Aircr. 27: 979-992

Lockenhauer J L, Layton G P 1976 RPRV research focus on HiMAT. Astronaut. Aeronaut. 14: 36-41

McCullers L A, Lynch R W 1974 Dynamic characterisation of advanced filamentary composite structures. Volume II. Aeroelastic synthesis procedure development. AFFDL-TR-73-111

Neill D J, Johnson E H, Canfield R 1990 ASTROS-A multidisciplinary automated structural design tool. J. Aircr. 27: 1021-1027

Noll T, Blair M, Cerra J 1986 ADAM, An aeroservoelastic method for analog or digital systems J. Aircr. 23: 852-858

Noll T E, Perry B, Gilbert M G 1989 Aeroservoelasticity: Current trends and future expectations. Australian Aeronautical Conference, Melbourne, pp. 176-180

Petiau C 1990 Structural optimisation of aircrafts-practice and trends. Proceedings of the 17th Congress of the Int. Council of the Aeronautical Sciences, Stockholm, Sweden, Paper No. ICAS-90-1.2.1 (New York: AIAA) 1: 210-221 
Sallee V J 1990 ADAM-2.0 - An ASE analysis code for aircraft with digital flight control systems. AIAA-90-1077-CP

Schneider G, Zimmermann H 1986 Static aeroelastic effects on high-performance aircraft. AGARD-R-725, pp. 2.1-2.15

Shirk M H, Hertz T J, Weisshaar T A 1986 Aeroelastic tailoring-theory, practice and promise. J. Aircr. 23: 6-18

Suryanarayan S, Panda K, Prakash B G, Sujata T 1992 Aeroservoelastic stability and response analysis: Integration of component analysis and software development. Report No. ADA/TD/STR/003, Aeronautical Development Agency, Bangalore

Upadhya A R, Ramani T S, Srinivasan K 1990a Approach to static aeroelastic analysis of a combat aircraft. Proc. Int. Conf. on Advances in Structural Testing, Analysis and Design (New Delhi: Tata-McGraw Hill)

Upadhya A R, Ramani T S, Srinivasan K, Rajasekaran P 1990b Studies on optimisation of an advanced fighter aircraft wing structure. Int. Conf. on Advances in Structural Testing, Analysis and Design (New Delhi: Tata-McGraw Hill)

Weisshaar T A, Foist B L 1982 Vibration and flutter of advanced composite lifting surfaces. AIAA paper 82-0722

Wilkinson K, Markowitz J, Lerner E, George D, Batill S M 1977 FASTOP: A flutter and strength optimisation program for lifting surface structures. J. Aircr. 14: 581-587 